\title{
Opening Editorial AIDS and Behavior Special Issue on Stigma
}

\author{
Kirk D. Henny ${ }^{1,2}$. Donna Hubbard McCree ${ }^{1}$ (D) Jonathan Mermin ${ }^{1}$
}

Accepted: 6 November 2021 / Published online: 14 January 2022

This is a U.S. government work and not under copyright protection in the U.S.; foreign copyright protection may apply 2021

\begin{abstract}
The papers in this Special Supplement provide insight into current research on and partnerships needed to address HIV-related stigma and better characterize the negative effects of HIV-related stigma on populations disproportionately affected by HIV in the United States. The findings may be used to inform evidence-based strategies and ideally additional interventional research with the goal of reducing stigma, new HIV infections, and improved health for persons with HIV.
\end{abstract}

Keywords HIV $\cdot$ Stigma $\cdot$ Interventions $\cdot$ Research $\cdot$ Disparities

\section{Background}

The year 2021 marks the fourth decade since the first five cases of what became known as the Acquired Immune Deficiency Syndrome (AIDS) were identified. The early days of the epidemic were marked by substantial morbidity and mortality, widespread stigma and discrimination, and few effective prevention and treatment options. However, because of progress in antiretroviral treatment, people with HIV (PWH) can have life expectancies close to people without the infection. In addition, there have been breakthroughs in prevention: PWH who are virally suppressed, i.e., have no detectable virus in their blood using routine nucleic acid tests, have effectively no risk of sexually transmitting the virus to their partners [1] and preexposure prophylaxis (PrEP) reduces the risk of acquiring HIV by over 99\% [2]. The availability of highly effective prevention and treatment options has led to a call for an end to the HIV epidemic [3]. This goal will not be realized, however, until the vast majority of PWH are aware of their status, and are virally suppressed, and persons who experience risk for infection have access to and use prevention options. Populations disproportionately affected by HIV in the United States experience social and structural

Kirk D. Henny

cso5@cdc.gov

1 National Center for HIV, Viral Hepatitis, STD and TB Prevention, Centers for Disease Control and Prevention, Atlanta, GA, USA

2 Division of HIV Prevention/NCHHSTP/CDC, 1600 Clifton Road NE, US8-4, Atlanta, GA 30329, USA barriers to prevention, treatment, and care, including stigma [4-7]. Stigma experienced by persons with HIV and persons from communities disproportionately affected by HIV has been associated with decreased access to and use of effective HIV prevention and treatment services and interventions. Consequently, the ultimate goal of ending the HIV epidemic cannot be fully realized without effective interventions and strategies to address and reduce HIV-related stigma.

HIV stigma is a multifaceted social process, wherein persons are socially devalued and often discriminated against due, in part, to their HIV status [8, 9]. HIV stigma often amplifies social inequities that affect access and uptake of critical resources to address an individual's HIV challenges [10]. External expressions of stigma may include social labeling, stereotyping or social discrimination [9, 11]. Social manifestations of stigma often lead to internalized stigma, wherein persons devalue themselves due to negative social connotations associated with HIV [11, 12]. Internalized HIV stigma is associated with negative outcomes affecting an individual's social well-being, mental health and social support [13, 14]. For many groups, HIV stigma intersects with other social factors (e.g., homophobia, racism) that consequently compound and reinforce the marginalization of individuals already coping with HIV [15]. Stigma thus exacerbates underlying factors that perpetuate and increase existing HIV-related disparities among disproportionately affected populations.

Barriers to accessing HIV-related services and negative health outcomes caused by HIV stigma have been well documented in the research literature. HIV stigma is correlated with individual behaviors that increase personal HIV risk. 
Specifically, persons experiencing HIV stigma report higher rates of condomless sex [16] and non-disclosure of HIV status to sex partners [17]. HIV-related stigma is also a barrier to receipt of HIV prevention services such as testing [18] and PrEP [19].

Studies indicate that HIV stigma can reduce uptake of essential HIV care services. For example, internalized stigma may contribute to depressive symptoms that lead individuals to reduce their engagement with HIV medical care services $[15,20]$. Further, greater internalized stigma is correlated with lower adherence to antiretrovirals among PWH [6, 21]. Ultimately, lower adherence negatively affects viral suppression causing worse health outcomes and increased transmission risk to sex partners [22].

A systemic review of studies focused on HIV-related stigma among healthcare providers revealed that clinicians with limited HIV-related stigma training are more likely to exhibit stigmatizing behavior toward patients [23]. Negative sequalae associated with HIV stigma manifest themselves across multiple dimensions of prevention and care services. However, there has been less research evaluating interventions that reduce HIV stigma, and no randomized studies directly evaluating the effect of stigma reducing actions on HIV incidence and related health disparities [24-26].

\section{Contribution of Special Issue Collection}

The papers in this volume provide insight into current research on and partnerships needed to address HIV-related stigma and better characterize the negative effects of HIVrelated stigma on populations disproportionately affected by HIV in the United States. Collectively, the findings may be used to inform evidence-based strategies and ideally additional interventional research with the goal of reducing stigma, new HIV infections, and improved health for persons with HIV.

Greenwood et al. summarized the HIV stigma research efforts and scientific findings across Institutes, Centers, and Offices (ICOs) at The National Institutes of Health. The focus is mission-related research gaps, priorities and/or future areas for research. Findings indicated a need for federal level prioritization of stigma research to inform the HIV public health field and achieve the goal of ending the HIV epidemic in the United States. Kalichman et al. described a novel approach, geometric conceptualization, for developing a psychometric instrument to measure intersectional enacted and anticipated stigma in a population of African American women with HIV. Results provide a first step towards measuring these concepts and identifying methods to address them in interventions and strategies to reduce stigma.

Beer et al. provided national estimates of HIV stigma and its associations with sociodemographic and clinical characteristics among person with diagnosed HIV. The findings suggest a need for addressing stigma around disclosure and community attitudes in interventions to reduce the negative effects of stigma on PWH. Further, Gunn et al. summarized results from a systematic review that quantified the effects of HIV stigma interventions for gay, bisexual and other men who have sex with men (MSM) on stigma and sex risk. Results show that including a stigma reduction component in interventions can improve HIV testing and reduce sex risk for MSM. The authors concluded that interventions to reduce stigma are important to decreasing HIV infection in this population.

Regarding partnerships, Valentine et al. described the importance of community engagement in decisions about the needs, barriers, including experiences with stigma, and opportunities for better sexual health prevention efforts to reduce sexually transmitted infections in rural geographic areas. Arnold et al. discussed the advantages of faith-based partnerships in efforts to address stigma and reduce HIVrelated disparities. Finally, Burnham Crockett et al. explored stigma experienced in healthcare settings as a barrier to ending the HIV epidemic. Results highlight the importance of engaging PWH in the development of interventions, the need for interventions in settings that do not specialize in HIV care, and the importance of engaging all staff when addressing HIV-related stigma in healthcare settings.

Considering the negative effects of stigma on disproportionately affected populations, Batchelder et al. assessed differences in reported discrimination related to HIV, race, sexual orientation, and substance use and internalized stigmas among MSM with HIV who use substances. The authors found that younger participants reported higher levels of all types of discrimination and internalized stigma; perceived community HIV stigma accounted for these associations. As such, the authors concluded that age, year of diagnosis, and race should be considered in interventions that address stigma for this population. Further, MeyersPantele et al. examined the effects of HIV stigma on mental health outcomes among sexual minority men (SMM) with HIV. Findings suggested that internalized stigma (i.e., the endorsement of negative thoughts and beliefs surrounding) and enacted stigma (i.e., experiences of discrimination, prejudice, or stereotyping), but not anticipated HIV stigma (i.e., the expectation that other people will treat you negatively in the future because of your HIV status) are important intervention targets for stimulant use, depression, and stress among SMM with HIV [27-29]. Daniel et al. examined the effects of intersecting identities, e.g., immigrant status, race/ ethnicity, and limited English proficiency on African immigrants. Results illuminated the critical need for communityled approaches to address HIV prevention efforts like HIV testing in this population. Finally, Watson et al. explored communication dynamics negatively affected by HIV stigma 
in an investigation among persons who inject drugs during the 2019 HIV outbreak in West Virginia.

Research literature has documented the negative effects of HIV stigma regarding access to and use of HIV-related services and contribution to negative health outcomes. Interventions have been designed to reduce stigma and improve mental health in PWH and communities disproportionately affected by HIV. However, few interventions have been developed to reduce stigma, while improving measurable health outcomes or HIV incidence. The special issue collection represents an effort to address such gaps and further expand the knowledge base related to HIV stigma.

Author Contributions All authors whose names appear on the submission: (1) made substantial contributions to the conception of the work; (2) drafted the work and revised it critically for important intellectual content; (3) approved the version to be published; and (4) agree to be accountable for all aspects of the work.

Funding There was no funding for this article.

\section{Declarations}

Conflict of interest All authors declare that they have no conflict of interest.

Ethical Approval This article does not contain any studies with human participants or animals performed by any of the authors.

Disclosure The findings and conclusions in this Editorial are those of the authors and do not necessarily represent the official positions of the Centers for Disease Control and Prevention.

\section{References}

1. Cohen MS, et al. Prevention of HIV-1 infection with early antiretroviral therapy. N Engl J Med. 2011;365(6):493-505.

2. Centers for Disease Control and Prevention. Effectiveness of prevention strategies to reduce the risk of acquiring or transmitting HIV. https://www.cdc.gov/hiv/risk/estimates/prevention strategies.html\#anchor_1562942347 (2019). Accessed 25 Oct 2021.

3. Fauci A, et al. Ending the HIV epidemic: a plan for the United States. JAMA. 2019;321(9):844-5.

4. Kemp C, et al. HIV stigma and viral load among AfricanAmerican women receiving treatment for HIV. AIDS. 2019;33(9):1511-9.

5. Seeley J, Blanc AK. Reducing stigma and discrimination: new evidence and its implications. AIDS. 2020;34(Suppl 1):S1-4.

6. Sweeney SM, Vanable PA. The association of HIV-related stigma to HIV medication adherence: a systematic review and synthesis of literature. AIDS Behav. 2016;20(1):29-50.

7. Williams R, et al. The relationship between individual characteristics and HIV-related stigma in adults living with HIV: medical monitoring project, Florida, 2015-2016. BMC Public Health. 2020;20(1):723.
8. Goffman E. Stigma: notes on the management of spoiled identity. New York: Touchstone; 1986.

9. Link BG, Phelan JC. Stigma and its public health implications. Lancet. 2006;367(9509):528-9.

10. Parker R, Aggleton P. HIV and AIDS-related stigma and discriminatin: a coceptual framework and implications for action. Soc Sci Med. 2003;57(1):13-24.

11. Earnshaw V, et al. HIV stigma and physical health symptoms: do social support, adaptive coping, and/or identity centrality act as resilience resources? AIDS Behav. 2015;19(1):41-9.

12. Christopoulos K, et al. Internalized HIV stigma predicts subsequent viremia in US HIV patients through depressive symptoms and ART adherence. AIDS. 2020;34:1665-71.

13. Bogart LM, et al. Perceived discrimination and mental health symptoms among Black men with HIV. Cult Divers Ethnic Minor Psychol. 2011;17(3):295-302.

14. Logie C, Gadalla TM. Meta-analysis of health and demographic correlates of stigma towards people living with HIV. AIDS Care. 2009;21(6):742-53.

15. Chambers LA, et al. Stigma, HIV and health: a qualitative synthesis of literature. BMC Public Health. 2015;15:848.

16. Burnham KE, et al. Trauma symptoms, internalized stigma, social support and sexual risk behavior among HIV-positive gay and bisexual MSM who have sought sex partners online. AIDS Care. 2016;28(3):347-53.

17. Smith R, Rossetto K, Peterson BL. A meta-analysis of disclosure of one's HIV-positive status, stigma and social support. AIDS Care. 2008;20(10):1266-75.

18. Boehme AK, et al. HIV-infected women's relationships with their health care providers in the rural deep south: an exploratory study. Health Care Women Int. 2012;33(4):403-19.

19. Cahill S, et al. Stigma, medical mistrust, and perceived racism may affect PrEP awareness and uptake in black compared to white gay and bisexual men in Jackson, Mississippi and Boston, Massachusetts. AIDS Care. 2017;29(11):1351-8.

20. Relf MV, et al. Discrimination, medical distrust, stigma, depressive symptoms, antiretroviral medication adherence, engagement in care, and quality of life among women living with HIV in North Carolina: a mediated structural equation model. J Acquir Immune Defic Syndr. 2019;81(3):328-35.

21. Katz IT, et al. Impact of HIV-related stigma and treatment adherence: systematic review and meta-synthesis. J Int AIDS Soc. 2013;16(3):18640.

22. Rodger AJ, et al. Sexual activity without condoms and risk of HIV transmission in serodifferent couples when the HIV-positive partner Is using suppressive antiretroviral therapy. JAMA. 2016;316(2):171-81.

23. Geter A, Herron AR, Sutton MY. HIV-related stigma by healthcare providers in the United States: a systematic review. AIDS Patient Care STDS. 2018;32(10):418-24.

24. Hatzenbuehler ML, Phelan JC, Link BG. Stigma as a fundamental cause of population health inequalities. Am J Public Health. 2013;103(5):813-21.

25. Brewer R, et al. An exploratory study of resilience, HIV-related stigma, and HIV care outcomes among men who have sex with men (MSM) living with HIV in Louisiana. AIDS Behav. 2020;24(7):2119-29.

26. Quinn K, et al. Multiple dimensions of stigma and health related factors among young black men who have sex with men. AIDS Behav. 2017;21(1):207-16.

27. Earnshaw VA, et al. HIV stigma mechanisms and well-being among PLWH: a test of the HIV stigma framework. AIDS Behav. 2013;17(5):1785-95.

28. Earnshaw V, Chaudoir S. From conceptualizing to measuring HIV stigma: a review of HIV stigma mechanism measures. AIDS Behav. 2009;13(6):1160-77. 
29. Turan B, et al. Framing mechanisms linking HIV-related stigma, adherence to treatment, and health outcomes. Am J Public Health. 2017;107(6):863-9.
Publisher's Note Springer Nature remains neutral with regard to jurisdictional claims in published maps and institutional affiliations. 